\title{
Rôle de la réponse immunitaire innée dans le cerveau
}

$>$ Nous savons depuis peu qu'il existe une réponse

Véronique Blais, Serge Rivest immunitaire innée dans le système nerveux central. Cette réponse s'amorce à partir des structures non protégées par la barrière hématoencéphalique et du réseau microvasculaire cérébral et s'étend progressivement à l'ensemble du cerveau au cours d'une infection systémique. L'activité transcriptionnelle des gènes codant pour les protéines de la réponse immunitaire innée est aussi fortement stimulée lors de différentes perturbations neurologiques. Cette découverte récente a soulevé l'hypothèse selon laquelle l'inflammation pourrait contribuer à la neurodégénérescence et à la démyélinisation. Les molécules immunitaires peuvent également stimuler la libération des facteurs neurotrophiques et, par conséquent, promouvoir la réparation et la remyélinisation des neurones. Ainsi, la réponse innée inflammatoire cérébrale peut, d'une part, protéger les neurones mais, d'autre part, être une cause directe de certaines maladies neurodégénératives. <

\section{Immunité innée}

L'immunité innée est la première ligne de défense des êtres vivants pour combattre les pathogènes et leurs toxines. Cette réponse met en œuvre des phénomènes locaux, comme l'accroissement du débit sanguin, l'augmentation de la perméabilité vasculaire et la migration des leucocytes par diapédèse, ainsi que des phénomènes systémiques, comme la sécrétion de protéines plasmatiques par le foie, l'élévation de la température corporelle et la mobilisation des substrats énergétiques. Le rôle primordial de cette réponse est d'éliminer les substances étrangères par phagocytose et de présenter ces antigènes aux cellules du système immu-

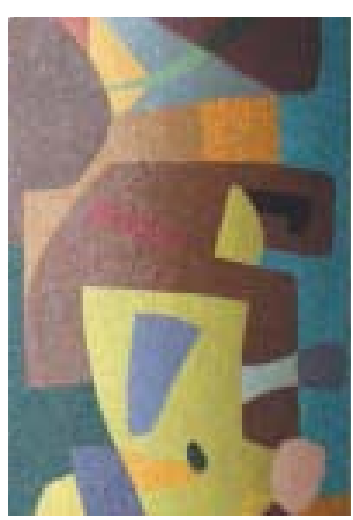

nitaire spécifique, les lymphocytes. Les phagocytes sont par conséquent les cellules clé du système immunitaire inné et les mécanismes de reconnaissance et d'activation de ces cellules présentatrices d'antigènes (CPA) ont été caractérisés au cours des dernières années.

En effet, la présence d'une infection est détectée par l'hôte grâce à la reconnaissance d'éléments spécifiques produits par les micro-organismes [1]. Ces éléments, appelés PAMP (pathogen-associated molecular patterns), sont reconnus par les CPA et amorcent la réponse innée. Un de ces PAMP, le lipopolysaccharide (LPS), constituant principal de la membrane externe des bactéries à Gram négatif, représente un exemple bien connu de reconnaissance innée conduisant à une réponse inflammatoire par les phagocytes [2]. D'autres PAMP, les peptidoglycanes et l'acide lipotéichoïque des bactéries à Gram positif, activent la voie de signalisation du NF-KB (nuclear factor $K B$ ) et la production de cytokines. La sécrétion de cytokines par les monocytes/neutrophiles et les macrophages tissulaires en 
réponse aux PAMP implique une cascade d'événements signalétiques cellulaires.

Dès sa découverte, la protéine Toll a été considérée comme une molécule essentielle au développement de l'axe dorsoventral de l'embryon de la drosophile. Les TLR (Toll-like receptors) sont les homologues de cette protéine chez les mammifères et ils sont exprimés à la surface des CPA. Ces cellules sont rapidement activées par les microorganismes qui lient un ou plusieurs membres de la famille des TLR de façon très spécifique. Les membres de cette famille de récepteurs partagent des domaines extracellulaires et cytoplasmiques similaires [3]. Leur domaine extracellulaire possède plusieurs motifs riches en leucines, tandis que leur domaine cytoplasmique est très semblable à la portion cytoplasmique du récepteur de type I de l'interleukine I (IL-IRI), communément appelé domaine TIR (Toll/IL1R) $[1,3,4]$. Des TLR distincts reconnaissent les principaux PAMP produits par les bactéries à Gram négatif ou à Gram positif. Le rôle du TLR4 dans la signalisation des bactéries à Gram négatif a été proposé, à l'origine, à partir du fait que les souris ayant des mutations dans le locus Lps sont totalement insensibles au LPS, et que le locus Lps code pour TLR4 $[5,6]$. En revanche, les souris dont le gène codant pour le TLR2 a été inactivé développent une réponse inflammatoire normale en présence du LPS [7], mais pas en présence des parois cellulaires de bactéries à Gram positif [7]. Ces résultats démontrent que TLR2 reconnaît de façon très stricte les éléments produits par les bactéries à Gram positif et que TLR4 lie les PAMP des bactéries à Gram négatif. D'autres TLR ont cependant la capacité de reconnaître des éléments communs des bactéries et des virus.

Huit autres membres de la famille des TLR sont aujourd'hui caractérisés. La flagelline bactérienne est reconnue par le TLR5 [8], tandis que le TLR9 est responsable de la réponse inflammatoire en présence d'ADN bactérien [9]. Les TLR amorcent aussi une réponse immune innée en présence d'ARN double-brin produit par certains virus. Ainsi, les souris dont le gène TLR3 a été inactivé ne répondent pas à l'acide polyinosine-polycytidylique qui est l'analogue synthétique de I'ARN double-brin [10]. De plus, les TLR peuvent former des complexes multimériques qui élargissent le spectre des molécules qu'ils reconnaissent. Ainsi, la dimérisation des domaines cytoplasmiques des TLR2 n'induit pas la production de cytokines par les macrophages, tandis que la dimérisation des domaines cytoplasmiques de TLR4 stimule l'activité NF-KB [11]. Le domaine cytoplasmique de TLR2 forme des hétérodimères fonctionnels avec TLR6 et/ou TLR1 [11].
Le domaine TIR des TLR et de I'IL-IRI interagit avec MyD88. Cette protéine adaptatrice possède un domaine de mort (death domain) amino-terminal qui s'associe à IRAK (IL-IR-associated kinase), ainsi qu'une une sérine kinase qui active une autre molécule adaptatrice, TRAF6 (tumor necrosis factor receptor-associated factor 6). Le recrutement de TRAF6 mène à l'activation de la protéine kinase IKK (IKB kinase), qui est composée de deux sous-unités catalytiques (IKK $\alpha$ et IKK $\beta$ ) et d'une sous-unité régulatrice (IKK $\gamma / N E M O)$ [12]. IKK $\gamma$ est nécessaire pour l'activation de NF-KB par plusieurs stimulus, bien que seule la sous-unité IKK $\beta$ soit essentielle pour l'activation de ce facteur de transcription par les cytokines et les PAMP. En effet, IKK $\beta$ est la cible des signaux en amont produits par les stimulus proinflammatoires qui mènent à la phosphorylation et à la dégradation d'|KB $[13,14]$. Le recrutement du complexe MyD88/IRAK/TRAF6 dans le domaine cytoplasmique du récepteur peut aussi activer la voie des kinases MAPK (mitogen-activated protein kinase), en particulier la voie de signalisation de la kinase JUN avec l'activation du facteur de transcription AP-1 (activator protein 1). L'activation de la transcription de la grande majorité des gènes qui prennent part à la réponse immunitaire innée est attribuable à la translocation nucléaire d'AP-1 et NF-KB.

\section{Réponse immune innée dans le système nerveux central}

Le cerveau a longtemps été considéré comme un organe privilégié d'un point de vue immunologique, puisque la barrière hémato-encéphalique (BHE) et ses jonctions serrées empêchent la transmigration des cellules du système immunitaire systémique. Toutefois, le cerveau possède son propre système de défense qui peut se mettre rapidement en état d'alerte à la moindre infection systémique. La réaction cérébrale s'amorce à partir des organes circumventriculaires (CVO) où les vaisseaux sanguins de ces organes n'ont pas de jonctions serrées semblables à celles qui caractérisent la BHE du tissu nerveux. Les bactéries et les PAMP peuvent donc diffuser librement à travers les organes circumventriculaires. Plusieurs récepteurs des PAMP sont aussi exprimés de façon constitutive dans les CVO et à la suite d'une simple injection systémique d'un LPS, la transcription des cytokines pro-inflammatoires est d'abord activée dans ces structures dépourvues de BHE $[15,16]$. Le LPS circulant entraîne lui aussi une forte augmentation de l'expression des gènes codant pour le CD14, le TLR2, plusieurs cytokines et chimiokines, ainsi que les protéines du système du complément [17-22] dans les 
régions adjacentes aux organes CVO et progressivement dans l'ensemble du cerveau.

Cette vague inflammatoire via des cellules microgliales du système nerveux central (SNC) reste toutefois surprenante, puisque l'accès des endotoxines circulantes au tissu cérébral est limité par la BHE. Des molécules solubles doivent par conséquent prendre le relais afin d'activer la population de cellules immunitaires du cerveau. Le TNF- $\alpha$ (tumor necrosis factor) est responsable de cette action et active la microglie parenchymale de manière paracrine lors de l'endotoxinémie. À l'instar du LPS circulant, une simple injection de TNF- $\alpha$ dans le ventricule latéral stimule l'activité transcriptionnelle des gènes inflammatoires dans les cellules microgliales du parenchyme cérébral [19]. L'inhibition de l'activité biologique de cette cytokine dans le SNC peut aller aussi jusqu'à abolir les effets du LPS systémique sur la réponse immunitaire innée cérébrale [19]. En résumé, les récepteurs transmembranaires CDI4 et TLR4 sont exprimés à la surface des cellules monocytaires installées dans les organes non protégés par la BHE. La formation du complexe LPS/TLR4/MyD88 amorce la signalisation proinflammatoire et l'activité transcriptionnelle des gènes, dont le TNF- $\alpha$. Ce dernier active en retour la signalisation NF-KB et la transcription de gènes dans les cellules microgliales adjacentes (Figure I). La production cérébrale de TNF- $\alpha$ est par conséquent essentielle à l'activation des cellules microgliales du parenchyme lors d'une endotoxinémie sévère. Cette réaction organisée et coordonnée des cellules phagocytaires propres au cerveau pourrait être déterminante dans la protection des différents éléments neuronaux au cours de l'infection.

\section{La double nature de l'immunité innée dans le système nerveux central}

Les rôles du TLR2, du TLR4 et du CD14 dans le SNC sont encore méconnus. L'expression constitutive du CD14 et du TLR2 dans les CVO et leur forte induction dans le parenchyme cérébral par le LPS circulant confortent I'hypothèse selon laquelle ces molécules jouent un rôle dans la protection des cellules neuronales contre les PAMP. Les macrophages et la microglie des CVO sont positionnés de façon stratégique pour répondre rapidement à l'endotoxine circulante ou aux bactéries; les microglies du parenchyme nerveux représentent pour leur part la population de cellules phagocytaires du cerveau disponibles en cas d'invasion. L'altération de la BHE durant les infections sévères et les traumatismes cérébraux permet la diffusion de molécules qui normalement n'auraient pas accès aux éléments du paren- chyme. Certes l'activation de la microglie peut mener rapidement à l'élimination des intrus, mais une activité soutenue des cellules microgliales peut avoir des effets néfastes pour certains éléments du cerveau. Ainsi, nous avons récemment observé une forte expression des gènes codant le TLR2 et le CD14 dans le cerveau de souris atteintes d'encéphalomyélite autoimmune expérimentale $(\varepsilon A \varepsilon)$, un modèle expérimental de sclérose en plaques [23]. Cette observation concorde avec le fait que cette maladie démyélinisante a une étiologie immunitaire et est associée à une stimulation chronique en rapport avec la production de molécules inflammatoires. En revanche, une induction similaire de TLR2 se manifeste après une lésion du cortex cérébral, une méningite virale et, de façon surprenante, chez des souris transgéniques qui surexpriment une forme mutante de la superoxyde dismutase 1 que l'on trouve dans la sclérose latérale amyotrophique [24]. Ces résultats démontrent que les gènes codant pour les protéines du système immunitaire inné ne sont pas seulement induits par les PAMP, mais également après une lésion du cerveau ou lors de maladies neurodégénératives. II faut mentionner que l'expression de ces gènes immunitaires ne précède pas nécessairement les phénomènes de neurodégénérescence, mais qu'elle est observée après un traumatisme ou après la mort de différentes populations cellulaires du cerveau. La réponse immunitaire innée pourrait donc bien être une conséquence plutôt qu'une cause des maladies du cerveau. Cette perspective dépend bien entendu du modèle expérimental.

Bien que le LPS puisse induire l'expression une grande variété de gènes immunitaires dans le cerveau, ce phénomène ne semble pas être néfaste pour les neurones [25]. Pourtant, des effets neurotoxiques causés par le LPS ont cependant été rapportés dans certaines études $[26,27]$. Ces différences peuvent s'expliquer en considérant les techniques utilisées pour déterminer la neurodégénérescence, l'âge des animaux, la dose d'endotoxine, la durée d'injection et la région ciblée. De fait, une simple injection de LPS dans l'hippocampe, le cortex ou la substance noire de rats adultes n'est toxique que pour les neurones de la substance noire [27]. Alors que la microglie contribue à l'élimination des pathogènes du SNC, il est possible que des populations particulières de neurones soient plus sensibles aux molécules inflammatoires et que les cellules microgliales et les facteurs qu'elles sécrètent soient néfastes pour certains neurones. Un tel effet peut être observé lorsque le tissu cérébral est exposé à de grandes concentrations de LPS ou d'autres PAMP, comme dans les cas de méningites bactériennes chez les jeunes enfants. II est alors intéressant de noter que les cerveaux expriment une 


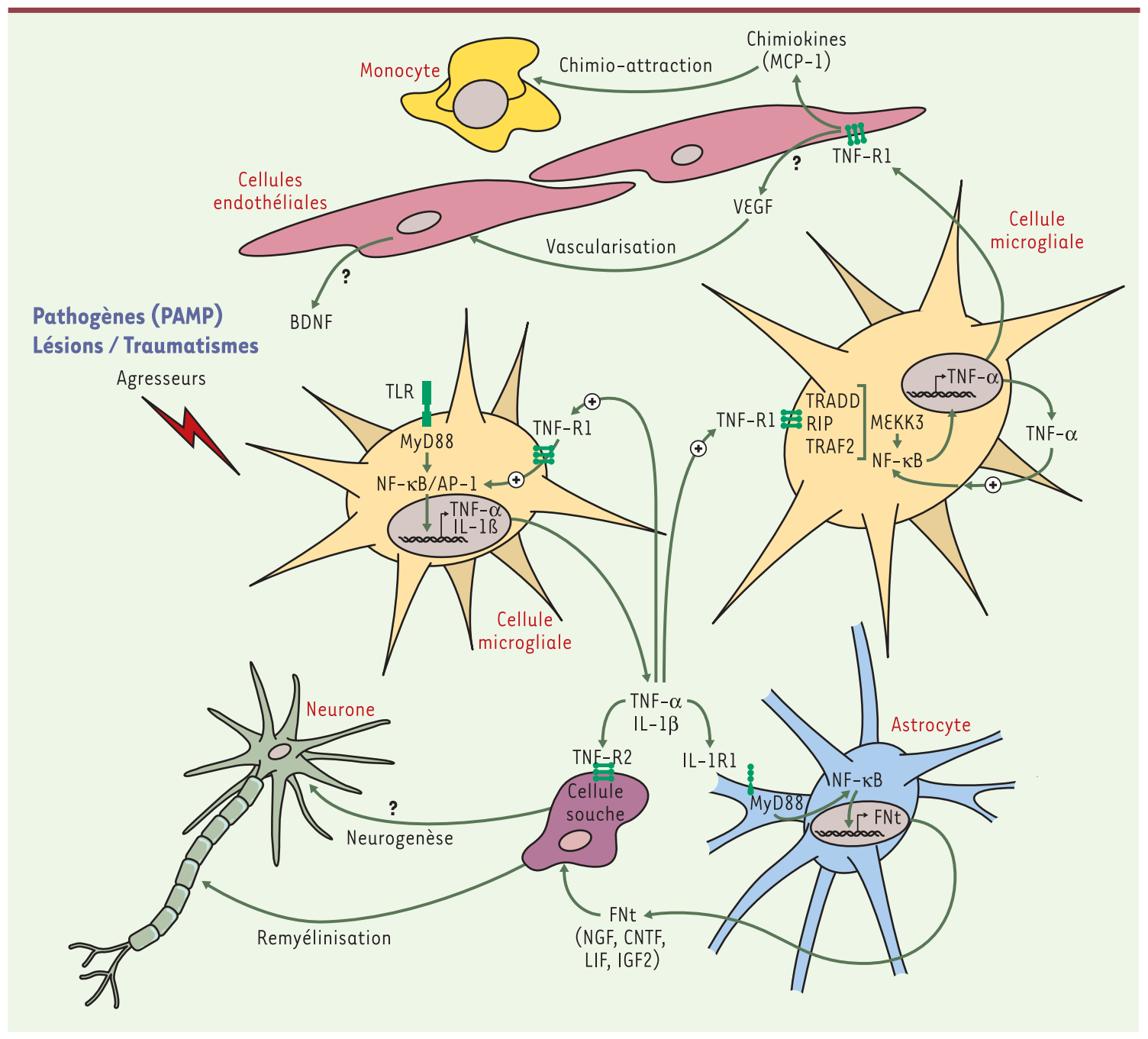

Figure 1. Rôles bénéfiques des cytokines pro-inflammatoires au cours de la réponse immune innée du cerveau. Les cellules microgliales sont stimulées par des pathogènes ou des traumatismes neurologiques, ce qui entraîne le recrutement de protéines adaptatrices dans le domaine intracellulaire des TLR (toll-like receptors). Des voies de signalisation pro-inflammatoires et la transcription de cytokines par les cellules présentatrices d'antigènes du cerveau sont alors activées. Le TNF- $\alpha$ (tumor necrosis factor $\alpha$ ) et I'IL-1 $\beta$ (interleukine-1 $\beta$ ) sont les deux principales cytokines de la réponse immune innée qui agissent sur les différentes cellules du système nerveux central. Le TNF- $\alpha$ agit de manière autocrine et paracrine sur les cellules microgliales qui expriment le récepteur de type 1 (TNF-RI). I'IL-1 $\beta$ stimule la production de facteurs neurotrophiques (FNt) astrocytaires par l'intermédiaire de son récepteur de type I (IL-1RI). Le CNTF (ciliary neurotrophic factor) et le NGF (nerve growth factor) agissent sur les progéniteurs pour faciliter la remyélinisation à la suite d'un traumatisme neurologique. L'interaction entre le TNF- $\alpha$ et son récepteur de type 2 (TNF-R2) peut également mener à la différenciation des cellules souches qui deviennent des oligodendrocytes matures. Bien que ce mécanisme ne soit pas encore bien démontré, il semble néanmoins que certaines molécules de la réponse inflammatoire pourraient stimuler la production de FNt, comme le VEGF (vascular endothelial growth factor) et le BDNF (brain-derived neurotrophic factor), par les cellules endothéliales. Or, le VEGF favorise la néovascularisation et induit la libération de BDNF qui contribue à la différentiation de certains types de neurones. En produisant des chimiokines, les cellules microgliales et endothéliales sont, enfin, capables d'attirer les cellules immunitaires provenant de la périphérie. PAMP: pathogen-associated molecular patterns; NF-KB: nuclear factor KB; LIF: leukemia inhibitory factor; IGF2: insulin-like growth factor 2; TRADD: TNFR1-associated protein with dead domain; TRAF: TNFR-associated factor; RIP: receptor-interacting protein; MEKK3: mitogen-activated protein kinase kinase kinase 3; MyD88: protéine adaptatrice MyD88 (adapté de [36]). 
plus grande susceptibilité à la réponse inflammatoire. Ainsi, en présence de LPS, le cerveau de jeunes rats de 7 jours est plus vulnérable à de courtes périodes d'hypoxie/ischémie qui, normalement, n'entraînent que très peu de dommages cérébraux [28].

Les effets bénéfiques de la réponse inflammatoire sont aussi multiples. Les cytokines pro-inflammatoires sont produites très rapidement par les cellules microgliales à la suite d'une lésion du tissu nerveux [29]. Le TNF- $\alpha$ et I'IL-1 $\beta$ sont les deux principales cytokines produites par la microglie du parenchyme adjacent au site lésionnel [29]. La sécrétion d'IL-1 $\beta$ par la microglie à pour effet de stimuler les astrocytes qui, en retour, produisent les facteurs neurotrophiques (FNt), dont le NGF (nerve growth factor) [30, 31], le CNTF (ciliary neurotrophic factor) [29] et I'IGF-1 (insulin-like growth factor 1) [32]. Les souris dont le gène codant pour l'IL-1 $\beta$ a été invalidé $\left(I L-1 \beta^{-/}\right)$présentent non seulement un retard important de différentiation des progéniteurs en oligodendrocytes, mais aussi un retard très significatif dans les processus de remyélinisation [32]. Ce problème de remyélinisation est en grande partie causé par l'absence de I'IL-1 $\beta$ microgliale qui joue un rôle majeur dans la stimulation des FNt par les astrocytes. Ces résultats démontrent que la production précoce d'IL-1 $\beta$ par les cellules microgliales du parenchyme est un mécanisme essentiel pour amorcer la libération des FNt par les astrocytes et faciliter la maturation des cellules souches du cerveau jusqu'au stade oligodendrocyte (Figure 1).

Le TNF et I'IL-1 ont de nombreux effets redondants dans le système immunitaire systémique. Toutefois, le TNF ne semble pas participer à la libération des FNt, du moins du CNTF [29]. La production de CNTF, qui est augmentée après une lésion du cortex cérébral, est complètement abolie chez les souris $I L-1 \beta^{-/}$, alors que l'expression de I'ARNm du TNF reste semblable à celle des souris sauvages [29]. L'IL-1 $\beta$ est en fait la cytokine stimulant la production des FNt par les astrocytes et le TNF, le facteur soluble agissant sur la microglie de manière autocrine et paracrine (Figure 1). En revanche, l'activation microgliale induite par le TNF peut être néfaste pour le cerveau en favorisant l'apoptose des oligodendrocytes et en empêchant la remyélinisation. Cette cytokine entraîne une résistance du récepteur de l'IGF-l dans les neurones et inhibe l'activation de la voie de signalisation de la phosphatidylinositol 3-kinase [33]. Malgré ce constat, plusieurs résultats contradictoires ont été relevés à propos du rôle joué par le TNF dans l'aggravation et/ou l'atténuation des dommages cérébraux. H.A. Arnett et al. [34] ont étudié les processus de démyélinisation et de remyélinisation chez des souris dont les gènes codant pour le TNF et ses récepteurs ont été invalidés. L'absence de TNF- $\alpha$ entraînait un important retard dans la remyélinisation, retard associé à une réduction du nombre de cellules progénitrices d'oligodendrocytes en prolifération et d'oligodendrocytes matures. Ces auteurs ont également signalé que les effets réparateurs du TNF- $\alpha$ sont attribuables au récepteur de type 2 (TNFR2), indiquant ainsi que le double rôle de cette cytokine, dans la démyélinisation et la remyélinisation, pourrait dépendre du type de récepteur en cause [34].

Le rôle du TNF et des autres cytokines dans la remyélinisation et la démyélinisation est cependant beaucoup plus complexe. Dans le modèle animal de la sclérose en plaques, les cellules T auxiliaires migrent dans le SNC et produisent du TNF et de l'interféron- $\gamma($ IFN $-\gamma)$. Un sousgroupe de cellules $T$ auxiliaires, les cellules $T_{H} l$ activées, et les cytokines qu'elles sécrètent, semblent jouer un rôle important dans l'étiologie de cette maladie. II est ainsi possible que le TNF- $\alpha$ et l'IFN- $\gamma$ agissent en synergie et entraînent des lésions des neurones et des autres cellules du SNC. Par exemple, I'IFN- $\gamma$ seul n'altère pas la survie cellulaire ou l'expression de la MBP (myelin basic protein) dans des cultures d'oligodendrocytes humains, mais rend ces cellules plus sensibles en présence de TNF [35]. De plus, I'IFN- $\gamma$ et le TNF- $\alpha$ accélèrent la dégénérescence en rapport avec l'administration intra-cérébrale d'un donneur de monoxyde d'azote (données non publiées). En revanche, une simple injection de TNF- $\alpha$ (avec ou sans IFN- $\gamma$ ) dans le cerveau de souris ou de rats provoque une forte réaction inflammatoire au niveau des cellules microgliales, mais n'induit pas de mort neuronale [19].

\section{Conclusions}

Il y a plusieurs raisons de croire que l'inflammation provoquée par les molécules de l'immunité innée soit un mécanisme à la fois néfaste, puisqu'il altère les neurones et les oligodendrocytes, et bénéfique, car il protège les cellules du cerveau contre les pathogènes. L'origine cellulaire des cytokines participant à la réaction inflammatoire provoquée par les molécules de l'immunité innée, ainsi que la nature de leurs récepteurs peuvent expliquer les résultats divergents des différentes études. La production chronique de protéines de l'immunité innée et la présence de cellules de l'immunité adaptative dans le cerveau semblent être des éléments favorisant la neurodégénérescence. En revanche, la réponse immune innée qui se manifeste rapidement après une infection systémique ou cérébrale n'est probablement pas préjudiciable au SNC. Une des 
conséquences bénéfiques de l'activité microgliale est la libération de FNt et d'autres molécules ayant des rôles importants dans le maintien de l'homéostasie du cerveau, la neuroprotection et la réparation cellulaire. Une réactivité microgliale persistante pourrait cependant être en cause dans la surproduction de molécules proinflammatoires et dans l'altération de la BHE. II a été démontré que ce mécanisme se manifestait dans plusieurs maladies neurodégénératives et démyélinisantes. Comme les cellules microgliales sont les cellules présentatrices d'antigènes du cerveau, elles jouent probablement un rôle crucial dans l'auto-immunité vis-à-vis des éléments neuronaux. Une meilleure compréhension de cette réponse immune innée dans le tissu cérébral devrait aider à éclaircir les mécanismes fondamentaux permettant au cerveau d'élaborer une réponse inflammatoire bénéfique, mais aussi nuisible, pour les neurones. $\Delta$

\section{SUMMARY}

Role of the innate immune response in the brain There is an innate immune system in the brain. It is inducible in a transient manner from the structures that are devoid of blood brain barrier and thereafter within parenchymal microglia during systemic infection. Transcriptional activation of genes encoding proteins of the innate immunity also takes place in diseases of the central nervous system. This recent discovery raised the hypothesis that inflammation and innate immunity may be involved in the etiology of neurodegenerative disorders. Nevertheless, this system is able to trigger the release of neurotrophic factors and to protect neuronal elements during brain infection and trauma. The innate immune response may play a critical role in protecting neurons and be a possible cause of neurodegeneration. The fate of this newly identified cascade of events is therefore likely to have a determinant impact on the central nervous system during infection and injury. $\Delta$

\section{RÉFÉRENCES}

1. Anderson KV. Toll signaling pathways in the innate immune response. Curr Opin Immunol 2000; 12: 13-9.

2. Wright SD. Toll, a new piece in the puzzle of innate immunity. J Exp Med 1999; 189: 605-9.

3. Akira S, Takeda K, Kaisho T. Toll-like receptors: critical proteins linking innate and acquired immunity. Nat Immunol 2001; 2: 675-80.

4. Muzio M, Polentarutti N, Bosisio D, Prahladan MK, Mantovani A. Toll-like receptors: a growing family of immune receptors that are differentially expressed and regulated by different leukocytes. J Leuk Biol 2000; 67: 450-6.

5. Beutler B. TIr4 : central component of the sole mammalian LPS sensor. Curr Opin Immunol 2000 ; 12: 20-6.

6. Poltorak A, He X, Smirnova I, et al. Defective LPS signaling in $\mathrm{C} 3 \mathrm{H} / \mathrm{HeJ}$ and C57BL/10ScCr mice: mutations in Tlr4 gene. Science 1998; 282 : 2085-8.

7. Takeuchi 0 , Hoshino K, Kawai $T$, et al. Differential roles of TLR2 and TLR4 in recognition of Gramnegative and gram-positive bacterial cell wall components. Immunity 1999; 11 : 443-51.

8. Hayashi F, Smith KD, Ozinsky $A$, et al. The innate immune response to bacterial flagellin is mediated by Toll- like receptor 5. Nature 2001; 410: 1099-103.

9. Hemmi H, Takeuchi 0, Kawai T, et al. A Toll-like receptor recognizes bacterial DNA. Nature $2000 ; 408: 740-5$

10. Alexopoulou L, Holt AC, Medzhitov R, Flavell RA. Recognition of doublestranded RNA and activation of NF-KB by Toll- like receptor 3 . Nature 2001 ; 413: 732-8.
11. Ozinsky A, Underhill DM, Fontenot JD, et al. The repertoire for pattern recognition of pathogens by the innate immune system is defined by cooperation between tolllike receptors. Proc Natl Acad Sci USA 2000; 97: 13766-71.

12. Chu WM, Ostertag D, Li ZW, et al. JNK2 and IKK $\beta$ are required for activating the innate response to viral infection. Immunity 1999; 11: 721-31.

13. Delhase M, Hayakawa M, Chen Y, Karin M. Positive and negative regulation of I $\mathrm{KB}$ kinase activity through IKK $\beta$ subunit phosphorylation. Science 1999; 284: 309-13.

14. Hu Y, Baud V, Delhase M, et al. Abnormal morphogenesis but intact IKK activation in mice lacking the IKK $\alpha$ subunit of IKB kinase. Science 1999; 284: 316-20.

15. Lacroix S, Feinstein D, Rivest S. The bacterial endotoxin lipopolysaccharide has the ability to target the brain in up regulating its membrane CD14 receptor within specific cellular populations. Brain Pathol $1998 ; 8: 625-40$.

16. Laflamme N, Rivest S. Tolllike receptor 4 : the missing link of the cerebral innate immune response triggered by circulating gramnegative bacterial cell wall components. FASEB J 2001 ; 15: 155-63.

17. Brochu S, Olivier M, Rivest S. Neuronal activity and transcription of proinflammatory cytokines, $\mid \kappa B \alpha$, and iNOS in the mouse brain during acute endotoxemia and chronic infection with Trypanosoma brucei brucei. J Neurosci Res 1999; 57: 801-16. 
18. Nadeau S, Rivest $S$. Regulation of the gene encoding tumor necrosis factor $\alpha$ (TNF- $\alpha)$ in the rat brain and pituitary in response in different models of systemic immune challenge. J Neuropathol Exp Neurol 1999; 58: 61-77.

19. Nadeau S, Rivest S. Role of microglial-derived tumor necrosis factor in mediating CDI4 transcription and nuclear factor $\kappa B$ activity in the brain during endotoxemia. J Neurosci 2000 ; 20: 3456-68.

20. Nadeau S, Rivest S. The complement system is an integrated part of the natural innate immune response in the brain. FASEB J 2001; 15: 1410-2.

21. Quan N, Whiteside M, Herkenham M. Time course and localization patterns of interleukin-1 $\beta$ messenger RNA expression in brain and pituitary after peripheral administration of lipopolysaccharide. Neuroscience 1998; 83: 281-93.

22. Thibeault I, Laflamme N, Rivest $\mathrm{S}$. Regulation of the gene encoding the monocyte chemoattractant protein 1 (MCP-1) in the mouse and rat brain in response to circulating LPS and proinflammatory cytokines. J Comp Neurol $2001 ; 434$ : 461-77.
23. Zekki H, Feinstein DL, Rivest $\mathrm{S}$. The clinical course of experimental

autoimmune encephalomyelitis is associated with a profound and sustained transcriptional activation of the genes encoding tolllike receptor 2 and CD14 in the mouse CNS. Brain Pathol 2002; 12: 308-19.

24. Nguyen MD, Julien JP, Rivest $S$. Induction of proinflammatory molecules in mice with amyotrophic lateral sclerosis: no requirement for proapoptotic interleukin$1 \beta$ in neurodegeneration. Ann Neurol 2001; $50: 630-9$.

25. Nadeau S, Rivest $S$. Endotoxemia prevents the cerebral inflammatory wave induced by intraparenchymal lipopolysaccharide injection: role of glucocorticoids and CDI4 J Immunol 2002; 169: 3370-81.

26. Castano A, Herrera AJ, Cano J, Machado A. Lipopolysaccharide intranigral injection induces inflammatory reaction and damage in nigrostriatal dopaminergic system. J Neurochem 1998 ; 70 : 1584-92.
27. Kim WG, Mohney RP, Wilson B, Jeohn GH, Liu B, Hong JS. Regional difference in susceptibility to lipopolysaccharideinduced neurotoxicity in the rat brain: role of microglia. J Neurosci 2000; 20: 6309-16.

28. Eklind S, Mallard C, Leverin $A L$, et al. Bacterial endotoxin sensitizes the immature brain to hypoxic-ischaemic injury. Eur J Neurosci 2001 ; 13: 1101-6.

29. Herx LM, Rivest S, Yong VW. Central nervous systeminitiated inflammation and neurotrophism in trauma: $I L-1 \beta$ is required for the production of ciliary neurotrophic factor. J Immunol 2000; 165: 2232-9.

30. Bandtlow C $E$, Meyer $M$, Lindholm D, Spranger $M$, Heumann R, Thoenen $H$. Regional and cellular codistribution of interleukin $1 \beta$ and nerve growth factor mRNA in the adult rat brain: possible relationship to the regulation of nerve growth factor synthesis. J Cell Biol 1990; 111: 1701-11.

31. DeKosky ST, Styren SD, O'Malley ME, et al. Interleukin-1 receptor antagonist suppresses neurotrophin response in injured rat brain. Ann Neurol 1996; 39: 123-7.
32. Mason JL, Suzuki K, Chaplin DD, Matsushima GK. Interleukin-1 $\beta$ promotes repair of the CNS. J Neurosci 2001; 21: 7046-52.

33. Venters HD, Tang Q, Liu Q, VanHoy RW, Dantzer R, Kelley KW. A new mechanism of neurodegeneration: a proinflammatory cytokine inhibits receptor signaling by a survival peptide. Proc Natl Acad Sci USA 1999; 96: 9879-84.

34. Arnett HA, Mason J, Marino M, Suzuki K, Matsushima GK, Ting JP. TNF $\alpha$ promotes proliferation of oligodendrocyte progenitors and remyelination. $\mathrm{Nat}$ Neurosci 2001; 4 : 1116-22.

35. Pouly S, Becher B, Blain M, Antel JP. Interferon- $\gamma$ modulates human oligodendrocyte susceptibility to Fasmediated apoptosis. J Neuropathol Exp Neurol $2000 ; 59: 280-6$.

36. Nguyen MD, Julien JP, RivestS. Innate immunity: the missing link in neuroprotection and neurodegeneration? Nat Rev Neurosci 2002 ; 3: 216-27.

\section{TIRÉS À PART}

S. Rivest 